\title{
Thermally stimulated exoelectron emission from solid Xe
}

\author{
I.V. Khyzhniy ${ }^{1}$, O.N. Grigorashchenko ${ }^{1}$, A.N. Ponomaryov ${ }^{2}$, E.V. Savchenko ${ }^{1}$, \\ and V.E. Bondybey ${ }^{2}$ \\ ${ }^{1}$ B. Verkin Institute for Low Temperature Physics and Engineering of the National Academy of Sciences of Ukraine \\ 47 Lenin Ave., Kharkov 61103, Ukraine \\ ${ }^{2}$ Lehrstuhl für Physikalische Chemie II TU München, 4 Lichtenbergstraße, Garching 85747, Germany
}

Received November 20, 2006

\begin{abstract}
Thermally-stimulated emission of exoelectrons and photons from solid Xe pre-irradiated by low-energy electrons were studied. A high sensitivity of thermally-stimulated luminescence (TSL) and thermally-stimulated exoelectron emission (TSEE) to sample prehistory was demonstrated. It was shown that electron traps in unannealed samples are characterized by a much broader distribution of trap levels in comparison with annealed samples and their concentration exceeds in number that in annealed samples. Both phenomena, TSL and TSEE, were found to be triggered by release of electrons from the same kind of traps. The data obtained suggest a competition between two relaxation channels: charge recombination and electron transport terminated by TSL and TSEE. It was found that TSEE predominates at low temperatures while at higher temperatures TSL prevails. An additional relaxation channel, a photon-stimulated exoelectron emission from pre-irradiated solid $\mathrm{Xe}$, was revealed.
\end{abstract}

PACS: 78.60.Kn Thermoluminescence; 79.75. + g Exoelectron emission.

Keywords: rare gas solids, thermally stimulated luminescence, exoelectron emission, relaxation processes.

\section{Introduction}

Relaxation processes in cryocrystals are of considerable interest from the standpoint of fundamental condensed-matter physics, and for various important applications especially for solid-state photochemistry. Elucidation of elementary relaxation stages in pre-irradiated solids can make a significant contribution to a controlled material modification via the electronic subsystem of the crystal. Exposure of solid insulating materials to ionizing radiation results in the modification of their physical properties. A number of structural defects, metastable species, ionic centers, trapped electrons, etc. are formed due to the interaction between the crystal and the radiation. Radiation-induced reactions produce guest atoms, molecules and radicals. Energy in different forms is stored by the crystal after the irradiation and can be released by heating or by irradiation with light initiating relaxation cascades. Electron traps play an important role in these processes. For a detailed understanding of the radiation effects it is necessary to follow the sequence of relaxation processes in solids after irradiation. Different classes of materials were investigated in view of this problem [1-3]. Rare gas solids (RGS) are effectively used as very convenient model systems for elucidating the elementary stages of charge transfer and energy relaxation in solid wide-gap insulators. These cryocrystals, composed of closed-shell atoms, possess comparatively simple electronic and crystal structure and weak interatomic forces in combination with a strong electron-lattice interaction. The specific properties of RGS give a possibility to understand electronically-induced processes on the atomic level (because of their high quantum yield) and to explain similar ones in more complex solids.

Activation spectroscopy methods are usually employed as powerful instruments for the analysis of the final stage of relaxation, e.g., processes occurring after the irradiation is completed. The method used most frequently is based on thermally stimulated luminescence (TSL) measurements. TSL of halogen- and oxygen-doped solid Xe was measured in [4] and [5] respectively. Kink at. al. [6] measured TSL of nominally pure solid Xe pre-irradiated by x-rays. In [7], the TSL was combined with vacuum ultraviolet spectroscopy methods to study creation of lattice defects via exciton self-trapping in solid Xe. 
Recombination of both charged and neutral centers contributes to the yield of TSL. As the temperature is increased neutral impurity atoms become mobile enough to move through the crystal and recombine, forming molecules in excited states. Radiative transitions of such molecules contribute to the TSL yield. It is difficult to distinguish this process, called chemiluminescence, from radiative recombination of charged particles using only the TSL method. That is why it is reasonable to perform simultaneous measurements of TSL and, for instance, thermally stimulated exoelectron emission (TSEE), e.g., apply TSL in combination with some kind of current activation spectroscopy methods. For this purpose we developed a low temperature modification of correlation spectroscopy, i.e., simultaneous measurements of TSL, TSEE and desorption of neutral atoms [8] from pre-irradiated RGS. In our previous experiments, TSEE was detected from solid $\mathrm{Ar}[9]$ and $\mathrm{Ne}[10,11]$. In this article we present our results of an activation spectroscopy study in solid Xe pre-irradiated with an electron beam.

\section{Experiment}

Xe cryocrystals were grown using a high purity gas (99.9996\%) were used. Before experiment the gas inlet system was pumped and degassed by heating under pumping. The samples were condensed from the gas phase under isobaric conditions $\left(P=10^{-7}\right.$ bar) on a metal substrate cooled by a closed-cycle 2-stage Leybold RGD 580 cryostat. The structure of the samples and therefore the distribution of the defect energy levels within the energy gap could be varied by changing the deposition temperature and the gas flux. The deposition rate was about $10^{-2} \mu \mathrm{m} / \mathrm{s}$. A typical sample thickness was $100 \mu \mathrm{m}$.

The samples were irradiated with electrons of $500 \mathrm{eV}$ at a current density of $30 \mu \mathrm{Acm}^{-2}$. The irradiation and recording of cathodoluminescence spectra were performed at low temperature $(6 \mathrm{~K})$ in order to exclude the conventional thermal mechanism of defect creation and to avoid the annealing of radiation-induced defects. The irradiation over TSL and TSEE yield spectra were recorded using different heating regimes. The total yield of TSL was measured with a PMT sensitized to VUV light.

In TSEE experiments the emission of electrons from pre-irradiated samples was detected with an Au-coated Faraday plate kept at a small positive potential $+9 \mathrm{~V}$. The current from the Faraday plate was amplified by a FEMTO DLPCA 100 current amplifier. The signal was reversed in polarity by an inverter and digitized in a PC.

For experiments on photon stimulated exoelectron emission we used a Coherent 899-05 dye laser pumped with Ar-ion laser. The power of laser beam was $35 \mathrm{~mW}$. The sample heating under laser light did not exceed $0.5 \mathrm{~K}$.

\section{Discussion}

After the irradiation with electrons the samples of RGS contain self-trapped holes, trapped electrons, metastable dopant states and other stable radiation-induced defects. To switch on the relaxation processes it is necessary to release electrons from their traps to the conduction band of the crystal. Because of the high mobility of free electrons in RGS they can move through the crystal and recombine with positive centers (intrinsic or extrinsic) giving rise to recombination luminescence. Note that holes are self-trapped in solid Xe as well as in other RGS. In Fig. 1 a correlation of the TSL and TSEE from the crystalline $\mathrm{Xe}$ is shown. The sample was deposited at $20 \mathrm{~K}$ to suppress the TSL and TSEE maxima in the temperature range lower then $20 \mathrm{~K}$.

The main maximum on the TSEE curve correlates with the first maximum in TSL while the main TSL maximum correlates with the high-temperature shoulder of the main TSEE peak. This means that TSL and TSEE are due to release of electrons from the traps characterized by the same activation energy. There is a competition between these thermally stimulated processes because electrons released from the traps have two possibilities - to recombine with positively charged centers or to escape from the surface of the crystal. It was found that at low temperatures TSEE predominates. As the temperature is increased, the probability of recombination reactions increases as evidenced by a rise of the the intensities of intrinsic and extrinsic recombination emission peaks in comparison with the TSEE yield.

Figure 2 represents a comparison of TSL and TSEE yields from the sample of solid Xe which was first annealed at $60 \mathrm{~K}$ and than irradiated with electrons. The main peak of TSEE appears at the same temperature as the high-temperature shoulder of the main TSL peak.

Taking into account the positive electron affinity of solid $\mathrm{Xe}(0.5 \mathrm{eV})$ [3], the peak of TSEE should be shifted

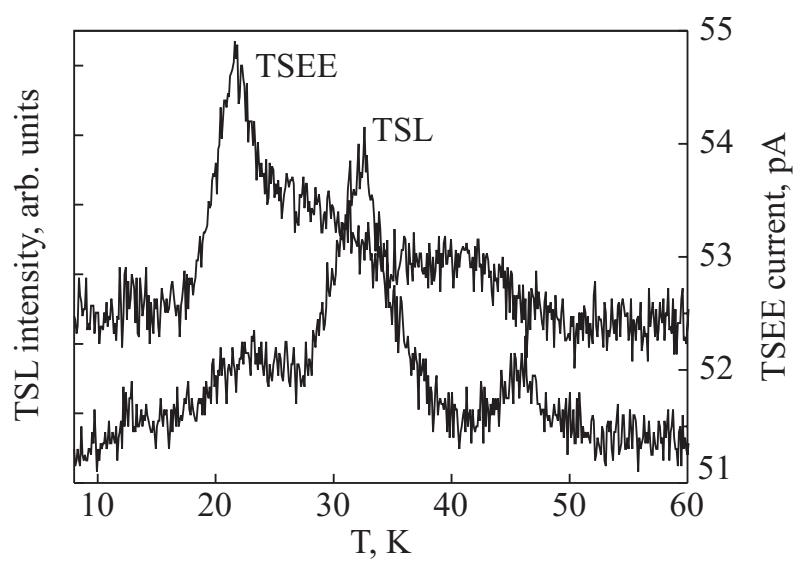

Fig. 1. A correlation of TSEE and TSL total yield from solid Xe. The sample was deposited at $20 \mathrm{~K}$. 


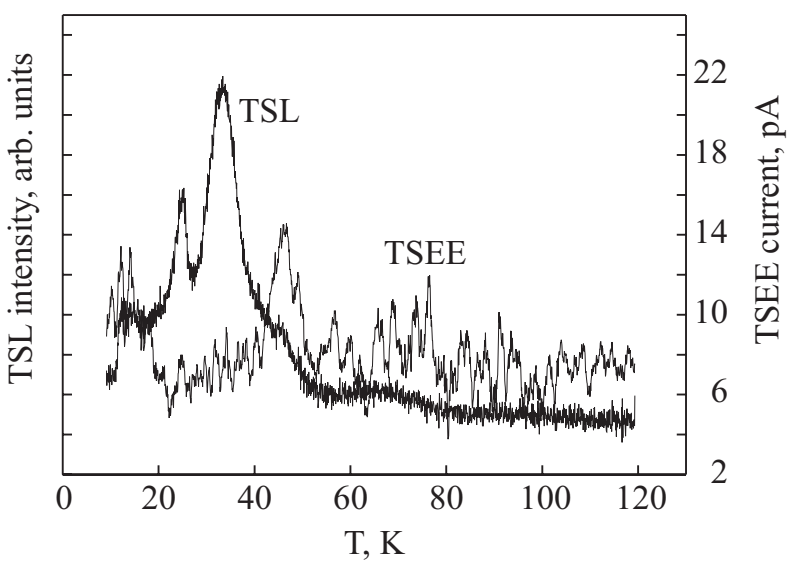

Fig. 2. Total yield of TSL and TSEE current from solid Xe annealed at $60 \mathrm{~K}$.

to higher temperatures in comparison with the corresponding TSL peak. We did not observe any shift. This could be caused by the negative space charge of accumulated in the sample during irradiation. However, this assumption needs further experimental verification.

Figure 3 demonstrates the effect of sample quality on TSL and TSEE. The samples annealed at temperatures close to the characteristic sublimation temperature contain fewer structural defects than the samples deposited at low temperatures without further annealing. Lattice de-
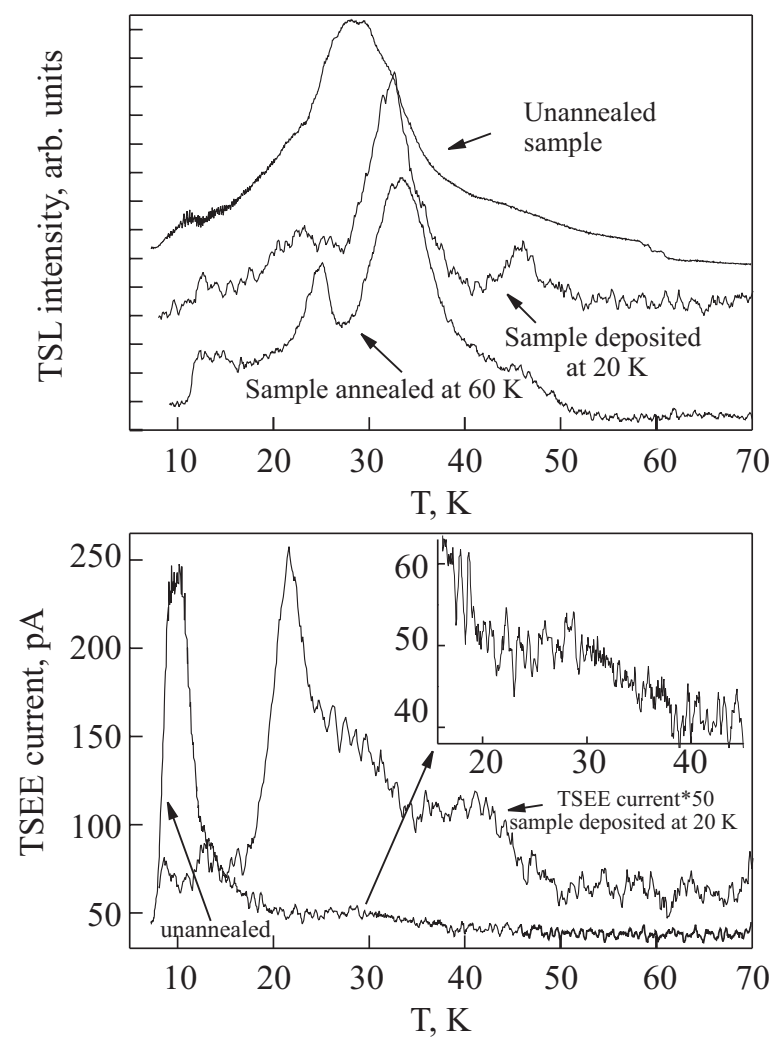

Fig. 3. Comparison of TSL and TSEE yields from the solid Xe samples of different quality. fects serve as shallow traps for electrons. The more defects in the crystal, the lower concentration of electrons escaping from traps at low temperatures and vice versa. That is the reason why the low-temperature peak predominates in TSEE from unannealed samples. The TSL spectrum in this case is a broad band of a few overlapping peaks. In the crystal grown at $20 \mathrm{~K}$ the maxima below this temperature are not observed either in TSL or TSEE because of a depopulation of traps with the corresponding activation energies formed during sample preparation. The structure of the TSL and TSEE curves in this case resolves.

Note that the values of TSEE current for the sample deposited at $20 \mathrm{~K}$ and for the one annealed at $60 \mathrm{~K}$ (Fig. 3) are multiplied by 50 and 100 , respectively, because they are much lower than those from the unannealed sample. The only pronounced feature in TSEE from the sample of solid Xe annealed at $60 \mathrm{~K}$ is the peak at about $45 \mathrm{~K}$. The low-temperature maxima are strongly suppressed.

Taking into account that RGS have comparatively wide conduction bands (about several eV), one can expect effective release of electrons under the beam of visible light both from deep and shallow traps with a subsequent cascade of relaxation processes. The photonstimulated exoelectron emission (PSEE) from pre-irradiated solid Xe is shown in Fig. 4. The initial decrease of the exoelectron emission current recorded just after switching off the electron beam (range 0-100 s) is the so-called afteremission caused by the presence of metastable guest atoms in the Xe matrix, i.e., nitrogen (from residual gases in the vacuum chamber). These centers, which are formed during the irradiation of the sample by electrons, exhibit a long afterglow after switching off the irradiating beam. This internal source of photons in the visible range causes the phenomenon of afteremission.

Figure 4 demonstrates the influence of visible range photons on the exoelectron emission from pre-irradiated solid Xe.

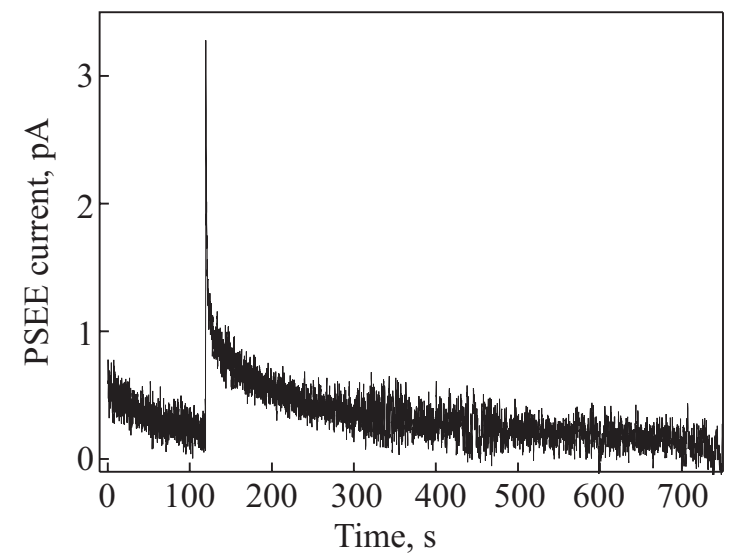

Fig. 4. Photo-stimulated exoelectron emission current from solid Xe. 
The laser light of $510 \mathrm{~nm}$ wavelength was directed on the sample surface when the afteremission was on the background level. We detected a strong increase of the exoelectron emission current. Then the current decayed exponentially in time because the electron traps were depopulating under the influence of the laser light. The initial part of the decay curve can be fitted by a first-order exponential function with the characteristic decay time $\tau=(15 \pm 4) \mathrm{s}$.

\section{Summary}

Relaxation processes in solid Xe pre-irradiated with an electron beam were studied by activation spectroscopy methods - thermally stimulated luminescence in combination with thermally- and photon-stimulated exoelectron emission measurements. Correlated in time measurements of TSL and TSEE from electron beam pre-irradiated solid Xe were performed for the first time. The main role of electron traps in the charge and energy transfer processes has been demonstrated. The channel relaxation related with the depopulation of traps induced by visible light has been revealed.

1. N. Itoh and A.M. Stoneham, Material Modification by Electronic Excitations, University Press, Cambridge (2000).
2. Ch.B. Lushchik and A.Ch. Lushchik, Decay of Electronic Excitations With Defect Formation in Solids, Nauka, Moscow (1989).

3. K.S. Song and R.T. Williams, Self-Trapped Excitons. Springer Series in Solid-State Science, Springer Verlag, Berlin (1996), v. 105.

4. M.E. Fajardo and V.A. Apkarian, J. Chem. Phys. 89, 4124 (1988).

5. A.V. Danilychev and V.A. Apkarian, J. Chem. Phys. 99, 8617 (1993).

6. M. Kink, R. Kink, V. Kisand, J. Maksimov, and M. Selg, Nucl. Instr. Meth. Phys. Res. B122, 668 (1997).

7. E.V. Savchenko, A.N Ogurtsov, I.V. Khyzhniy, G. Stryganyuk, and G. Zimmerer, Phys. Chem. Chem. Phys. 7, 785 (2005).

8. E.V. Savchenko and V.E. Bondybey, Phys. Status Solidi A202, 221 (2005).

9. E.V. Savchenko, O.N. Grigorashchenko, A.N. Ogurtsov, V.V. Rudenkov, G.B. Gumenchuk, M. Lorenz, A. Lammers, and V.E. Bondybey, J. Low Temp. Phys. 122, 379 (2001).

10. O.N. Grigorashchenko, V.V. Rudenkov, I.V. Khyzhniy, E.V. Savchenko, M. Frankowski, A.M. Smith-Gicklhorn, M.K. Beyer, and V.E. Bondybey, Fiz. Nizk. Temp. 29, 1147 (2003) [Low Temp. Phys. 29, 876 (2003)].

11. M. Frankowski, E.V. Savchenko, A.M. Smith-Gicklhorn, O.N. Grigorashchenko, G.B. Gumenchuk, and V.E. Bondybey, J. Chem. Phys. 121, 1474 (2004). 